\title{
Efeito do treinamento com videogames ativos nas dimensões morfológica e funcional: estudo clínico randomizado
}

\author{
Effects of active video games training in the morphological and functional \\ dimensions: randomized clinical trial
}

\author{
Raphael José Perrier-Melo1* ${ }^{*}$, Jorge Luiz Brito-Gomes², Nuno Domingos Garrido ${ }^{3,4}$, Saulo \\ Fernandes Oliveira ${ }^{5}$, Fernando José de Sá Pereira Guimarães ${ }^{6}$, Manoel da Cunha Costa ${ }^{7}$
}

ARTIGO ORIGINAL | ORIGINAL ARTICLE

\begin{abstract}
O objetivo do presente estudo foi analisar o efeito de programas de treinamento com videogames ativos (VGAs) estruturados e não estruturados sobre a composição corporal e desempenho nos testes de aptidão física durante seis semanas. Participaram do estudo 20 homens, os quais foram divididos homogeneamente em três grupos: Grupo controle - GC ( $n=7 ; 20.6 \pm 2.8$ anos), Grupo Experimental Estruturado -GET ( $n=6$; $20.2 \pm 2.1$ anos) e Grupo Experimental Não estruturado GEN ( $n=7 ; 19,1 \pm 1,2$ anos). O programa com VGAs foi realizado três sessões por semana (30 minutos cada) durante seis semanas. Houve melhoras significativas nos testes de apoio de frente (GET $p=0.02$ ), flexibilidade (GEN: $p=0.04$ e GET: $p=0.01$ ) e preensão manual (GEN: $\mathrm{p}=0.05)$. E apenas o GEN aumentou significativamente o percentual de gordura $(p=0.05)$ e massa gorda $(p=0.01)$. Conclui-se que após seis semanas de treinamento com VGAs, os dois grupos experimentais apresentaram manutenção na massa magra e aumento no nível de aptidão física. Palavras-chave: exercício; atividade física; jogos de vídeo; composição corporal; aptidão física.
\end{abstract}

ABSTRACT

The aim of this study was to analyze the effect of training programs with active video games (AVGs) structured and unstructured on body composition and performance in the physical fitness tests during six weeks. Study participants were 20 men who were evenly divided into three groups: Control Group - CG (n $=7 ; 20.6 \pm 2.8$ years), Experimental Structured Group -GET $(n=6 ; 20.2 \pm 2.1$ year) and Experimental Unstructured Group - GEN ( $n=7 ; 19.1 \pm 1.2$ years). The AVGs program was performed three times a week (thirty minutes each) during six weeks. There were significant improvements in the front support testing (GET $p=0.02)$, flexibility (GEN: $p=0.04$ and GET: $p=0.01)$ and handgrip $(G E N p=0.05)$. And only GEN increased significantly the fat percentage $(p=0.05)$ and fat mass $(p=0.01)$. After six weeks of each parallel AVGs training, these two groups presented maintenance in lean mass and increase in the level of physical fitness.

Keywords: exercise; physical activity; videogames; body composition; physical fitness.

Artigo recebido a 04.09.2015; Aceite a 22.03.2016

${ }^{1}$ Universidade de Pernambuco, Recife, Brasil. Escola Superior de Educação Física, ESEF, Brasil.

${ }^{2}$ Universidade Federal de Pernambuco, Vitória de Santo Antão, Brasil. Centro Acadêmico de Vitória, CAV, Brasil.

${ }^{3}$ Universidade de Trás-os-Montes e Alto Douro, Vila Real, Portugal.

${ }^{4}$ Centro de Investigação em Desporto, Saúde e Desenvolvimento Humano, CIDESD, Portugal

${ }^{5}$ Universidade Federal de Pernambuco, Vitória de Santo Antão, Brasil. Centro Acadêmico de Vitória, CAV, Brasil.

${ }^{6}$ Universidade de Pernambuco, Recife, Brasil. Escola Superior de Educação Física, ESEF, Brasil.

${ }^{7}$ Universidade de Pernambuco, Recife, Brasil. Escola Superior de Educação Física, ESEF, Brasil.

* Autor correspondente: Universidade de Pernambuco, Rua Arnóbio Marques, 310, Santo Amaro, RecifePernambuco, Brasil. E-mail: rperrier2@gmail.com 


\section{INTRODUÇÃO}

A inatividade física associada as atividades sedentárias de lazer tais como jogar videogame, utilizar computador e assistir televisão, aumentam o screen time - tempo de tela das pessoas, diretamente relacionado ao aumento dos fatores de riscos cardiovasculares e diminuição da aptidão física (Davies, Vandelanotte, Duncan, \& Uffelen, 2012). A manutenção desses comportamentos sedentários à longo prazo, favorecem o desenvolvimento e/ou agravamento das doenças crônicas degenerativas não transmissíveis e outras morbidades, como sobrepeso, obesidade, diabetes e hipertensão (Chau et al., 2014; Grøntved et al., 2014). Por outro lado, a prática regular de atividade física/exercício favorece na prevenção e tratamento dessas doenças, além de produzir benefícios relacionados à aptidão física e qualidade de vida (Garber et al., 2011).

É possível tornar uma pessoa fisicamente ativa por meio da realização de atividade física/exercício de forma planejada, estruturada e repetida com nível de intensidade leve a moderada, segundo o recomendado pelo American College of Sports Medicine - ACSM (Haskell et al., 2007). Dessa maneira, diversos métodos de atividades físicas eletrônicas têm sido propostos com o objetivo de manter ou otimizar um ou mais componentes da aptidão física relacionada à saúde, bem como as capacidades físicas de força/resistência muscular e flexibilidade (Pate, 1988; Peng, Crouse, \& Lin, 2012).

Os programas de treinamento com videogames ativos (VGAs), estruturados (jogos específicos que seguem os princípios do treinamento para avanço no desempenho) e não estruturados (jogos esportivos-recreativos que não seguem os princípios do treinamento), surgem como uma forma de atividade física, na qual proporciona diferentes estímulos à musculatura esquelética, alterando os diversos componentes da aptidão física relacionada à saúde (Lieberman et al., 2011).

Estudos anteriores (Adamo, Rutherford, \& Goldfield, 2010; Warburton et al., 2007) reportam que programas de treinamento com VGAs, quando realizados entre seis e dez semanas, oferecem melhoras em torno da aptidão física relacionada à saúde. No entanto, ainda são poucas as evidências científicas que verificaram o tempo mínimo necessário para provocar tais alterações na população adulta jovem. Neste sentido, considerando que os VGAs são categorizados como uma forma de atividade física e que proporcionam modificações positivas na composição corporal e incrementos de força, resistência muscular localizada e flexibilidade, torna-se necessário investigar os efeitos da sua prática sistematizada sobre a manutenção ou desenvolvimento dos marcadores da aptidão física e composição corporal.

Sendo assim, o objetivo deste estudo foi analisar o efeito de programas de treinamento com VGAs (estruturado e não estruturado) sobre a composição corporal e desempenho nos testes de aptidão física durante seis semanas de intervenção. A hipótese do estudo foi que o programa com VGA estruturado, no qual possui características semelhantes do treinamento físico, apresentaria melhores valores e alterações precoces sobre os testes da aptidão física e alterações na composição corporal.

\section{MÉTODO}

Trata-se de um estudo clínico randomizado UTN: U1111-1159. No qual recebeu aprovação do comitê de ética da instituição local (protocolo $n^{\circ}$ 858.209), respeitando a Resolução 244/2012 do Conselho Nacional de Saúde.

\section{Amostra}

A determinação mínima dos sujeitos foi calculada pelo programa estatístico Gpower versão 3.0, respeitando um poder amostral $>80 \%$ sobre um nível de significância de $5 \%$ (erro alfa: 0,05). A análise indicou que seria necessário o mínimo de 18 participantes (6 em cada grupo)

A amostra foi constituída por universitários, do sexo masculino, com idade entre 18 e 25 anos, os quais foram recrutados de maneira voluntária, por meio de rede social e cartazes distribuídos pela Universidade local, no ano de 2014. Os critérios de inclusão para participação do estudo foram: I) ser adulto jovem (18 aos 25 anos), do sexo masculino; II) não fazer parte de algum 
programa de exercício físico há pelo menos 3 meses; III) não apresentar comprometimento físico (articular ou muscular) e/ou audiovisuais; IV) responder negativamente ao Questionário de Prontidão para a Atividade Física (PAR-Q) e V) não ser usuário frequente de videogames ativos. Foram excluídos: I) os que faltaram em mais de $15 \%$ das sessões; II) os sujeitos que adquiriram algum comprometimento físico que impedissem ou contraindicassem a realização das atividades propostas e/ou III) os que iniciaram programas de exercício físico.

Após os critérios de seleção 24 voluntários foram recrutados para o estudo. Durante o programa de treinamento quatro participantes foram excluídos: três por não completarem a frequência mínima necessária e um em virtude de complicações musculares fora do ambiente de pesquisa. Dessa forma, a amostra final consistiu em 20 homens adultos jovens, os quais completaram mais de $85 \%$ das sessões de treino com VGA.

\section{Delineamento experimental}

Primeira etapa

Após o critério de seleção da amostra, os sujeitos incluídos foram encaminhados ao Laboratório de Avaliação e receberam informações gerais a respeito dos procedimentos a serem adotados nas coletas e foram orientados a assinar o Termo de Consentimento Livre e Esclarecido.

\section{Anamnese}

A triagem inicial consistiu na realização de uma anamnese, com o intuito de unir informações relacionadas aos aspectos sócio demográficos (nome, idade e sexo), além da confirmação de ausência dos fatores de risco cardiovasculares, obtidos pela mensuração da pressão arterial e do preenchimento negativo do PAR-Q. Em seguida, foram orientados a retornar ao laboratório no dia seguinte para a realização da bateria de avaliações.

\section{Instrumentos e Procedimentos}

Todas os procedimentos foram realizados no mesmo local e por um único avaliador treinado.
Os voluntários foram encaminhados a realização das medidas antropométricas, do exame de absorciometria radiológica de dupla energia (DEXA), avaliação antropométrica do somatório das dobras cutâneas (DC), teste de resistência muscular localizada, força de preensão manual, flexibilidade e um teste incremental máximo em cicloergometro.

Os sujeitos foram aconselhados a manter o padrão alimentar e horário do sono, a comparecer ao estudo vestidos de maneira adequada para avaliação e a não transportarem objetos metálicos para não causar interferência nos resultados da DEXA. Foi também recomendado a não realização de atividades físicas exaustivas nas 24 horas precedentes, absterem-se do consumo de produtos que contivessem cafeína, álcool ou tabaco nas três últimas horas antes dos testes e foram orientados a urinar e evacuar antes das avaliações.

\section{Antropometria e Composição Corporal}

As variáveis massa corporal e estatura foram mensuradas obedecendo as técnicas da International Society for the Advancement of Kinanthropometry - ISAK (Stewart, MarfellJones, Olds, \& Ridder, 2001). Utilizou-se uma balança (Filizola, Brasil), com precisão de 100g e um estadiômetro de madeira montado, com escala em milímetros para a verificação da estatura. O Índice de Massa Corporal (IMC) foi calculado pela massa corporal $(\mathrm{kg})$ dividida pela estatura (m) ao quadrado.

Para determinar o somatório das dobras cutâneas foi realizado a mensuração da espessura da dupla camada de gordura + pele nas regiões do tríceps, subescapular, bíceps, axilar média, peitoral, supra ilíaca, abdominal, coxa e perna, utilizou-se o adipômetro da marca Lange (EUA), com precisão de $0.1 \mathrm{~mm}$. Coletou-se duas medidas, caso apresentassem diferenças, seria realizada a terceira, adotando a mediana como valor final. Para avaliação do percentual (\%) de gordura total, massa magra e massa gorda, foi realizado o exame de absorciometria radiológica de duplo feixe - DEXA (Hologic, Discovery WI). 


\section{Testes Neuromotores}

Para o teste de resistência muscular abdominal, os sujeitos permaneceram deitados em decúbito dorsal, com as pernas flexionadas, joelhos formando um ângulo de $90^{\circ}$, com os pés fixados ao chão (auxílio do avaliador) e mãos cruzando os ombros. Em seguida, mediu-se o número máximo de flexões abdominais durante sessenta segundos. Para avaliar a resistência muscular localizada de apoio de frente, os voluntários partiram de uma posição inicial em quatro apoios, com o corpo estendido e cotovelos flexionados, em seguida realizavam uma extensão completa dos cotovelos, voltando à posição inicial. Foi verificado o número máximo de execuções realizadas de maneira correta independentemente do tempo de acordo com o protocolo estabelecido por Pollock e Wilmore (1993).

Para avaliação da flexibilidade, utilizou-se o teste de sentar e alcançar com o aparelho banco de Wells. Os sujeitos mantiveram-se sentados em frente ao banco de Wells, permanecendo com as pernas estendidas e os calcanhares encostados no equipamento. Com as mãos sobrepostas, inclinaram o tronco sobre o banco deslizando pela régua, até atingir o máximo alcance mantendo os joelhos estendidos (Pollock \& Wilmore, 1993).

O desempenho na força de preensão manual em $\mathrm{kg}$, foi verificada por meio do dinamômetro (Jamar, EUA), no qual considerou-se o maior valor registrado em duas medidas (Heyward, 2004).

\section{Avaliação Metabólica}

Os voluntários foram submetidos à uma análise direta do consumo de oxigênio (CPX/D, Cortex, Germany) com máscara (Metalyser, Germany), por meio de um teste incremental máximo no cicloergômetro de frenagem eletromagnética (Cateye, Japão) de acordo com o protocolo descrito por Astrand Ryhming (1954). Em seguida foram alocados de maneira homogênea, de acordo com os valores dos resultados obtidos no teste incremental máximo nos grupos: Grupo Experimental não Estruturado - GEN, Grupo Controle - GC e Grupo Experimental Estruturado - GET.
Videogames utilizados e grupo controle

O GET utilizou o jogo Microsoft Nike Kinect Fitness ${ }^{\circledR}$, trata-se de uma atividade estruturada, que possui características semelhantes às do treinamento físico, voltada para melhora da performance, por meio de um trabalho personalizado. $\mathrm{O}$ jogo utiliza exercícios calistênico, envolvendo movimento de saltar, empurrar, mudança de direção, agachamento, corrida estacionária, push-ups e abdominais. Além disso, conta com a realização de uma avaliação inicial, com o objetivo de classificar o estado de condicionamento físico atual do participante, e direcioná-lo para as atividades.

O GEN fez o uso do Microsoft Kinect Sports ${ }^{\circledR}$ Boxe, que é um jogo baseado em uma disputa de boxe, com o objetivo de vencer o adversário, como na luta real. É um jogo de característica livre - não estruturado, no qual o participante tem até três Rounds para definir a partida, e obrigatoriamente realiza movimentos laterais, rotações e saltos para se defender e atacar, aumentando a movimentação corporal e a motivação.

Diferente dos grupos experimentais o grupo controle (GC) não realizou atividades físicas durante o período de intervenção, permanecendo com suas atividades cotidianas normais.

\section{Segunda etapa - Protocolo de treinamento}

$O$ protocolo de treinamento consistiu em três sessões semanais, com duração de 30 minutos por sessão. Cada grupo realizou um programa de treinamento com videogame ativo, com as atividades virtuais do console Microsoft Kinect $X$ box $360^{\mathrm{TM}}$, durante um período de seis semanas, baseado nas diretrizes do ACSM (2007) e de estudos realizados anteriormente (Adamo et al., 2010; Lee \& Shin, 2013; Warburton et al., 2007).

As sessões experimentais foram realizadas, em horários alternados (manhã e tarde), de acordo com a disponibilidade dos sujeitos, mantendo intervalo de um dia entre elas. Antes das partidas, os voluntários eram orientados a seguir tais condutas: não realizar atividades exaustivas nas 24 horas antecedentes, abster-se de produtos que contivessem cafeína, álcool ou tabaco nas últimas três horas e eram 
aconselhados a realizar refeições leves 30 minutos à uma hora antes das sessões.

Durante o período de intervenção, a progressão do treinamento foi garantida apenas para o GET, pois a cada quatro semanas a programação dos exercícios e estímulos eram modificados pelo próprio sistema. Já o GEN apesar de manter a mesma intensidade (nível: amador), era possível elevar a velocidade de reação do oponente durante a partida. Porém, sem maiores dificuldades que garantissem uma variação no nível do jogo.

A estratégia adotada para verificar as possíveis evoluções nas variáveis estudadas a partir da avaliação inicial, foi a realização semanal (todas segundas-feiras) das medidas antropométricas e os testes neuromotores. Diferente das outras medidas, a DEXA foi realizada apenas na primeira e última avaliação.

\section{Análise Estatística}

Foi utilizada análise estatística descritiva e os dados estão apresentados em média \pm desviopadrão (DP). A normalidade da distribuição dos dados foi verificada por meio do teste de ShapiroWilk. Para examinar a relação intergrupos no momento pré intervenção, foi realizado o teste ANOVA one way. Para comparação intergrupo das variáveis antropométricas e dos testes da aptidão física nos momentos de avaliação foi utilizada análise de variância de dois caminhos para medidas repetidas, quando verificado efeito significativo, empregou-se o post-hoc de Bonferroni. Quanto as respostas pré e pós intervenção da composição corporal, utilizou-se $\mathrm{o}$ teste t-student pareado. Foi considerado significativo o valor de $\mathrm{p}<0.05$ (SPSS, versão 10.0).

\section{RESULTADOS}

$\mathrm{Na}$ Tabela 1, estão expostas as características demográficas, antropométricas, da composição corporal e metabólica da amostra. Não foram verificadas diferenças significativas nestas variáveis analisadas no início do estudo entre os grupos, podendo ser considerada uma amostra homogênea.

$\mathrm{Na}$ Tabela 2, estão apresentados os resultados das modificações ocorridas nas variáveis antropométricas e da composição corporal durante o programa de treinamento com VGAs estruturados e não estruturados. Pode-se notar que tanto o grupo experimental estruturado (GET), como o controle (GC) não demonstraram modificações significativas nessas variáveis. Por outro lado, o grupo experimental não estruturado (GEN) apresentou aumento significativo da massa gorda total $(\mathrm{p}=0.05)$ e do percentual de gordura total $(p=0.01)$.

A figura 1 apresenta os valores referentes ao desempenho nos testes de aptidão física durante os momentos de avaliação (momento inicial, durante e após seis semanas). A análise de variância com medidas repetidas não identificou diferenças significativas entre os grupos nos momentos de avaliação para todos os testes de aptidão física ( $\mathrm{p}>0.05)$.

Tabela 1

Característica antropométrica, da composição corporal e metabólica dos grupos no momento pré intervenção $(n=20)$.

\begin{tabular}{lccc}
\hline \multicolumn{1}{c}{ Variável } & GC $(\mathrm{n}=7)$ & GET $(\mathrm{n}=6)$ & GEN $(\mathrm{n}=7)$ \\
\hline Idade $(\mathrm{anos})$ & $20.6 \pm 2.8$ & $20.2 \pm 2.1$ & $19.1 \pm 1.2$ \\
IMC $\left(\mathrm{kg} / \mathrm{m}^{2}\right)$ & $22.4 \pm 2.6$ & $23.8 \pm 2.7$ & $23.9 \pm 2.5$ \\
Percentual de gordura total $(\%)$ & $22.0 \pm 2.3$ & $20.1 \pm 4.2$ & $22.7 \pm 3.8$ \\
Massa gorda total $(\mathrm{kg})$ & $15.3 \pm 2.8$ & $14.3 \pm 4.5$ & $17.0 \pm 4.8$ \\
Massa livre de gordura total $(\mathrm{kg})$ & $69.5 \pm 8.2$ & $69.9 \pm 9.0$ & $73.8 \pm 10.6$ \\
$\mathrm{VO}_{2}\left(\mathrm{ml} / \mathrm{kg} / \mathrm{min}^{-1}\right)$ & $36.9 \pm 4.5$ & $39.0 \pm 5.9$ & $36.0 \pm 5.1$ \\
\hline
\end{tabular}

IMC-Índice de Massa Corporal; \% - percentual; $\mathrm{VO}_{2}$ - Consumo de oxigênio; $\mathrm{n}$ - amostra. $\mathrm{p}<0.05$. 
75 | RJ Perrier-Melo, JL Brito-Gomes, ND Garrido, SF Oliveira, FP Guimarães, MC Costa

Tabela 2

Características antropométricas e da composição corporal dos grupos nos momentos pré, durante e pós intervenção.

\begin{tabular}{|c|c|c|c|c|c|c|c|c|c|c|c|}
\hline \multirow[b]{2}{*}{ Variável } & & \multirow[b]{2}{*}{$\begin{array}{l}\text { Idade } \\
\text { (anos) }\end{array}$} & \multicolumn{3}{|c|}{ Medidas antropométricas } & \multicolumn{3}{|c|}{ Composição corporal (DEXA) } & \multicolumn{3}{|c|}{ Somatório de dobras cutâneas (DC) } \\
\hline & & & $\begin{array}{c}\text { Massa } \\
\text { corporal }(\mathrm{kg})\end{array}$ & $\begin{array}{l}\text { Estatura } \\
(\mathrm{m})\end{array}$ & $\begin{array}{c}\text { IMC } \\
\left(\mathrm{kg} / \mathrm{m}^{2}\right)\end{array}$ & $\begin{array}{l}\text { Percentual de } \\
\text { gordura (\%) }\end{array}$ & $\begin{array}{l}\text { Massa gorda } \\
\text { total }(\mathrm{kg})\end{array}$ & $\begin{array}{l}\text { Massa magra } \\
\text { total }(\mathrm{kg})\end{array}$ & $\begin{array}{c}\sum \mathrm{DC} \\
\text { Central }\end{array}$ & $\begin{array}{c}\sum \mathrm{DC} \\
\text { Periférica }\end{array}$ & $\sum$ DC Total \\
\hline \multirow{3}{*}{$\begin{array}{c}\text { Pré } \\
\text { intervenção }\end{array}$} & GC & $20.6 \pm 2.8$ & $71.1 \pm 8.2$ & $1.7 \pm 0.6$ & $22.5 \pm 2.7$ & $22.0 \pm 2.3$ & $15.3 \pm 2.8$ & $51.6 \pm 5.6$ & $56.6 \pm 14.3$ & $38.9 \pm 7.2$ & $95.4 \pm 19.3$ \\
\hline & GET & $20.2 \pm 2.1$ & $71.2 \pm 8.8$ & $1.7 \pm 0.6$ & $23.1 \pm 2.3$ & $20.1 \pm 4.2$ & $14.3 \pm 4.5$ & $53.0 \pm 5.5$ & $49.0 \pm 15.5$ & $34.9 \pm 9.8$ & $83.9 \pm 25.0$ \\
\hline & GEN & $19.1 \pm 1.2$ & $75.5 \pm 10.7$ & $1.8 \pm 0.5$ & $23.9 \pm 2.5$ & $22.7 \pm 3.8$ & $17.0 \pm 4.8$ & $54.2 \pm 6.3$ & $62.7 \pm 18.7$ & $42.0 \pm 15.6$ & $104.7 \pm 33.2$ \\
\hline \multirow{3}{*}{$2^{\mathrm{a}}$ semana } & GC & - & $71.4 \pm 8.3$ & - & $22.6 \pm 2.7$ & - & - & - & $57.1 \pm 15.5$ & $38.9 \pm 7.5$ & $96.0 \pm 20.6$ \\
\hline & GET & - & $70.6 \pm 8.7$ & - & $22.9 \pm 2.2$ & - & - & - & $47.8 \pm 15.7$ & $34.8 \pm 10.7$ & $82.7 \pm 26.0$ \\
\hline & GEN & - & $75.2 \pm 10.4$ & - & $23.8 \pm 2.5$ & - & - & - & $61.7 \pm 19.0$ & $42.6 \pm 15.2$ & $104.3 \pm 32.8$ \\
\hline \multirow{3}{*}{$3^{\mathrm{a}}$ semana } & GC & - & $71.1 \pm 8.3$ & - & $22.5 \pm 2.7$ & - & - & - & $56.9 \pm 14.0$ & $38.7 \pm 7.6$ & $95.6 \pm 18.9$ \\
\hline & GET & - & $71.0 \pm 8.7$ & - & $23.0 \pm 2.1$ & - & - & - & $46.8 \pm 13.6$ & $35.3 \pm 10.8$ & $82.1 \pm 24.1$ \\
\hline & GEN & - & $75.4 \pm 11.0$ & - & $23.9 \pm 2.7$ & - & - & - & $61.1 \pm 19.0$ & $42.9 \pm 15.4$ & $104.0 \pm 33.2$ \\
\hline \multirow{3}{*}{$4^{\mathrm{a}}$ semana } & GC & - & $71.1 \pm 8.6$ & - & $22.5 \pm 2.8$ & - & - & - & $55.5 \pm 14.8$ & $39.1 \pm 9.3$ & $94.6 \pm 21.3$ \\
\hline & GET & - & $71.0 \pm 8.7$ & - & $23.0 \pm 2.1$ & - & - & - & $47.0 \pm 14.6$ & $33.5 \pm 9.1$ & $80.5 \pm 23.6$ \\
\hline & GEN & - & $75.4 \pm 10.6$ & - & $23.9 \pm 2.6$ & - & - & - & $60.9 \pm 19.1$ & $42.1 \pm 16.0$ & $103.0 \pm 34.2$ \\
\hline \multirow{3}{*}{$5^{\mathrm{a}}$ semana } & GC & - & $71.1 \pm 8.8$ & - & $22.5 \pm 2.8$ & - & - & - & $55.4 \pm 15.2$ & $39.3 \pm 7.8$ & $94.7 \pm 21.1$ \\
\hline & GET & - & $71.3 \pm 8.9$ & - & $23.1 \pm 2.2$ & - & - & - & $45.3 \pm 13.8$ & $33.3 \pm 9.9$ & $78.7 \pm 23.6$ \\
\hline & GEN & - & $75.7 \pm 10.7$ & - & $24.0 \pm 2.6$ & - & - & - & $60.9 \pm 19.3$ & $42.6 \pm 16.0$ & $103.4 \pm 34.5$ \\
\hline \multirow{3}{*}{$6^{\mathrm{a}}$ semana } & GC & - & $70.9 \pm 8.9$ & - & $22.4 \pm 2.9$ & - & - & - & $55.4 \pm 14.6$ & $39.3 \pm 8.0$ & $94.7 \pm 20.4$ \\
\hline & GET & - & $70.8 \pm 8.5$ & - & $22.9 \pm 2.1$ & - & - & - & $45.0 \pm 13.3$ & $33.0 \pm 9.7$ & $78.0 \pm 22.8$ \\
\hline & GEN & - & $75.9 \pm 10.4$ & - & $24.1 \pm 2.6$ & - & - & - & $60.4 \pm 19.5$ & $41.9 \pm 16.7$ & $102.3 \pm 35.2$ \\
\hline \multirow{3}{*}{$\begin{array}{c}\text { Pós } \\
\text { intervenção }\end{array}$} & GC & - & $71.1 \pm 9.0$ & - & $22.5 \pm 2.9$ & $22.0 \pm 2.3$ & $15.4 \pm 3.0$ & $51.7 \pm 6.1$ & $55.4 \pm 16.1$ & $38.9 \pm 8.4$ & $94.3 \pm 22.8$ \\
\hline & GET & - & $70.9 \pm 8.5$ & - & $23.0 \pm 2.0$ & $20.1 \pm 4.6$ & $14.1 \pm 4.5$ & $52.8 \pm 5.5$ & $44.0 \pm 13.6$ & $31.9 \pm 9.5$ & $75.9 \pm 22.9$ \\
\hline & GEN & - & $75.7 \pm 9.9$ & - & $24.0 \pm 2.4$ & $24.5 \pm 2.9^{*}$ & $18.3 \pm 4.3^{*}$ & $53.3 \pm 5.6$ & $58.6 \pm 20.5$ & $41.9 \pm 17.0$ & $100.4 \pm 36.5$ \\
\hline
\end{tabular}

DEXA - Exame de Absorciometria Radiológica de Duplo Feixe; $\sum$ DC - Somatório de Dobras Cutâneas; GC - Grupo Controle; GET - Grupo Experimental Estruturado; GEN - Grupo Experimental não Estruturado; IMC - Índice de Massa Corporal; $\sum$ DC central - peitoral, axilar, subescapular, suprailíaca e abdominal; $\sum$ DC periférica - bíceps, tríceps, perna e coxa média; * diferença significativa em relação ao repouso (teste t-student pareado). 
A figura 1 apresenta os valores referentes ao desempenho nos testes de aptidão física durante os momentos de avaliação (momento inicial, durante e após seis semanas). A análise de
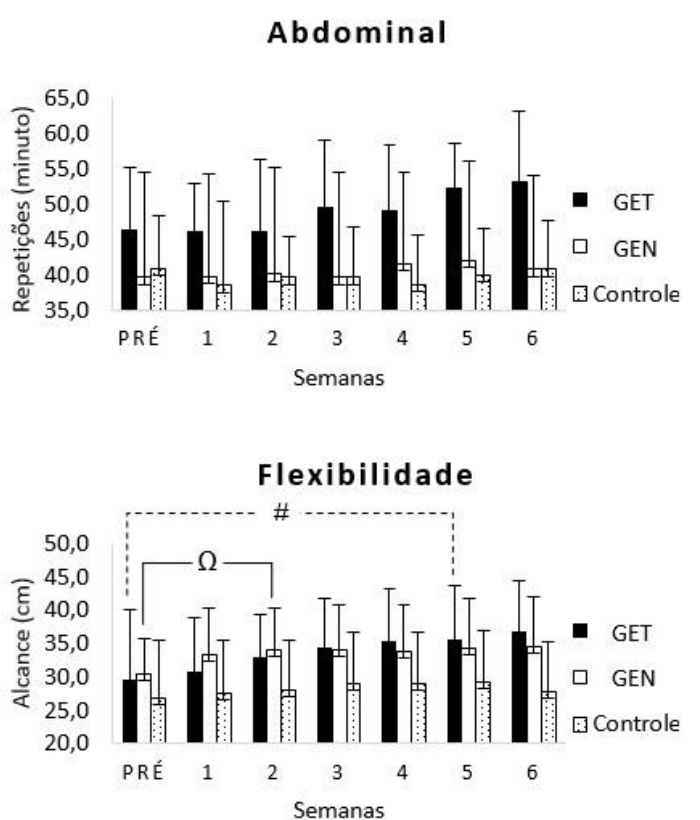

variância com medidas repetidas não identificou diferenças significativas entre os grupos nos momentos de avaliação para todos os testes de aptidão física ( $\mathrm{p}>0.05)$.
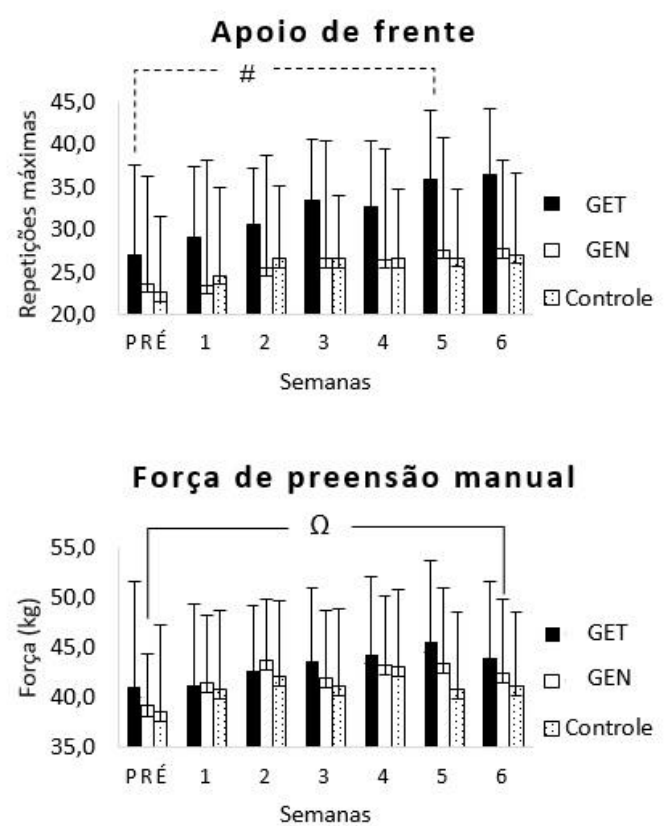

Figura 1. Desempenhos nos testes de aptidão física dos grupos nos momentos pré, durante e pósintervenção. \# diferença significativa entre os momentos pré e durante intervenção no GET (medidas repetidas); $\Omega$ diferença significativa entre os momentos pré e durante intervenção no GEN (medidas repetidas).

Pode-se observar que os grupos do presente estudo não apresentaram modificações significativas no teste de resistência muscular localizada de abdominal. Porém, a análise comparativa da variável de resistência muscular localizada de apoio de frente demonstrou que o grupo experimental estruturado - GET, obteve diferenças significativas entre os momentos pré e quinta semana de intervenção $(\mathrm{p}=0.02)$.

Ao analisar o desempenho no teste de flexibilidade pela análise intragrupo, verificou-se que o grupo experimental não estruturado GEN, obteve incrementos significativos entre o momento pré e segunda semana de intervenção $(\mathrm{p}=0,04)$. Já o grupo experimental estruturado GET, atingiu resultados significativos apenas entre o primeiro e quinto momento $(\mathrm{p}=0.01)$. Em relação a força de preensão manual, exclusivamente $\mathrm{o}$ grupo experimental não estruturado exibiu modificação significativa, entretanto, essa alteração só foi verificada ao final do programa de treinamento, entre o momento pré e pós intervenção $(\mathrm{p}=0.05)$.

\section{DISCUSSÃO}

O principal objetivo deste estudo foi analisar o efeito de seis semanas de intervenção com VGAs estruturados e não estruturados sobre a aptidão física e composição corporal de adultos jovens. Considerando que não houve diferenças significativas iniciais entre os grupos, confirmase a hipótese de que o treinamento com VGA estruturado apresentaria melhores resultados sobre a aptidão física e composição corporal. No entanto, não se confirma sobre a velocidade precoce do aparecimento das alterações, uma vez que apenas um teste (apoio de frente) apresentou melhoras significativas de maneira precoce no grupo estruturado.

Do nosso conhecimento, a presente pesquisa foi a primeira que realizou avaliações semanais durante os programas de treinamento com VGAs, com o intuito de identificar o momento que ocorre alterações sobre as variáveis de composição corporal e aptidão física relacionada à saúde. Neste sentido, os resultados indicam que períodos inferiores à seis semanas de 
intervenção são adequados para causar manutenção na massa magra total e ganhos significativos na força, resistência muscular localizada e flexibilidade nos grupos experimentais.

Observa-se na literatura a existência de estudos apresentando o efeito positivo do treinamento com VGAs sobre a diminuição dos componentes de gordura corporal (Adamo et al., 2010; Maddison et al., 2011). Entretanto, os resultados encontrados na presente pesquisa demonstram efeitos antagônicos, pois o grupo não estruturado aumentou significativamente os valores de massa gorda e \% de gordura, ao comparar os valores entre os momentos pré e pós intervenção, corroborando os resultados de Graves, Ridgers, Atkinson, e Atinkson (2010), que após 12 semanas de intervenção com VGAs exibiram aumento significativo na gordura corporal.

Outros estudos (Graves et al., 2010; Grilo, 1994; Machado et al., 2013) apontam que quando analisados isoladamente, os programas de exercício físico sem modificação na dieta e acompanhamento nutricional, não apresentaram resultados satisfatórios na diminuição significativa da gordura corporal. Em contrapartida, observa-se que a prática de exercício físico eleva o gasto energético e a necessidade de repor essas calorias após o treinamento, causando equilíbrio energético (Meirelles \& Gomes, 2004). Em nosso estudo o grupo não estruturado não alterou o nível de intensidade (amador) durante o programa de intervenção, diferente do grupo estruturado. Isso pode ter influenciado na manutenção do gasto energético e aumento do consumo calórico, atingindo um balanço energético positivo, auxiliando no acúmulo de gordura corporal (Drewnowski, Mennella, Johnson, \& Bellisle, 2012; Mendonça \& Anjos, 2004).

Com relação a força de preensão manual, os resultados mostram que o grupo estruturado não apresentou alteração significativa, corroborando os resultados obtidos no estudo de Warbuton et al. (2007). Entretanto, verifica-se que essa variável parece não ter sido influenciada pelo aumento da massa gorda e percentual de gordura. Pois de acordo com os painéis da figura 1 , o grupo
VGA não estruturado exibiu valores significativamente maiores entre os momentos pré e sexta semana, isto pode ter ocorrido devido a atividade realizada (jogo de boxe) utilizar predominantemente exercícios dinâmicos para os membros superiores, influenciando o aumento de impulsos excitatórios que favorecem maior recrutamento de unidades motoras, gerando maior nível de força (Maior \& Alves, 2003; Rodrigues, Pagnussat, \& Chiquetti, 2012).

Observou-se no presente estudo aumento significativo da flexibilidade em todos os grupos experimentais. Estes resultados são contrários aos achados no estudo de Warburton et al. (2007), que após seis semanas de intervenção com o VGA estruturado Gamebike, não identificaram alterações nesta variável. No entanto, nesta investigação o programa de treinamento não estruturado apesar de não possuir exercícios específicos para flexibilidade, obteve aumento significativo já na segunda semana de intervenção, diferente do grupo estruturado, o qual apresentou incrementos significativos apenas na quinta semana.

Provavelmente a manutenção da massa magra, bem como a execução de movimentos dinâmicos específicos, tais como agachamentos, socos e mudanças de direção, tenham sido aspectos que ocasionaram aumento do estímulo articular, refletindo em uma maior adaptação sensorial das propriedades musculotendíneas, influenciado assim o aumento da extensibilidade precoce dos sujeitos (ACSM, 1998; Gama, Medeiros, Dantas, \& Souza, 2007; Santos \& Domingues, 2008).

Quanto aos testes de resistência muscular localizada (RML), apesar do grupo estruturado apresentar média maior, não foram verificadas diferenças significativas entre os grupos experimentais durante as seis semanas de intervenção. Por outro lado, os dados apontam que a prática do VGA estruturado proporcionou incrementos significativos no teste apoio de frente pela análise intragrupo já na quinta semana.

Podemos destacar algumas possíveis explicações para apenas o GET ter apresentado progressos na RML. Uma delas está relacionada a característica mecânica do jogo, que utiliza 
princípios do treinamento físico, empregando o próprio corpo como carga externa, que de acordo com Pollock e Wilmore, (1993), exercícios quando realizados frequentemente com carga externa, proporcionam manutenção ou evolução da função muscular esquelética, causando eficiência no desempenho dos testes neuromotores. Além disso, uma vez que a massa magra apresenta certa manutenção nos valores ao longo do tempo, pode-se esperar efeito positivo no desempenho e ganho de resistência muscular (Lohman et al., 2008).

Uma das limitações do estudo foi a utilização apenas de homens para compor a amostra. Além disso, não houve controle da dieta e dos turnos das sessões de treino dos sujeitos. Porém, em locais de práticas de atividades físicas ou em ambientes residenciais, constantemente os praticantes não mantém uma regularidade no turno de treinamento, bem como no controle da ingestão calórica. Aumentando assim a validade externa deste presente estudo sobre a população estudada e os videogames ativos praticados nas residências em geral.

\section{CONCLUSÃO}

Conclui-se que após seis semanas de intervenção, com frequência de três dias por semana, o VGA estruturado proporcionou mudanças positivas na força de membro superior e flexibilidade. Já o VGA não estruturado, apesar de apresentar aumento nos componentes de gordura corporal, obtiveram evoluções em dois componentes da aptidão física (flexibilidade e força de preensão manual). Adicionalmente, percebemos que para a flexibilidade e para a resistência muscular localizada, a prática de VGAs pode proporcionar melhoras em períodos mais curtos do que seis semanas de treinamento. Sendo assim, os VGAs são eficientes para manutenção e desenvolvimento da aptidão física em adultos previamente não treinados.

Agradecimentos:

Nada a declarar

\section{Conflito de Interesses:}

Nada a declarar.

\author{
Financiamento: \\ Coordenação de Aperfeiçoamento de Pessoal de Nível \\ Superior (Capes)
}

\section{REFERÊNCIAS}

ACSM, American College of Sports Medicine Position Stand (1998). The recommended quality and quantity of exercise for developing and maintaining cardiorespiratory and muscular fitness and flexibility in healthy adults. Medicine and Science in Sports and Exercise, 30(6), 975991.

Adamo, K. B., Rutherford, J. a, \& Goldfield, G. S. (2010). Effects of interactive video game cycling on overweight and obese adolescent health. Applied Physiology, Nutrition, and Metabolism, 35(6), 805-15. http://doi.org/10.1139/H10-078

Astrand, P. O., \& Ryhming, I. . (1954). A Nomogram for Calculation of Aerobic Capacity (Physical Fitness) From Pulse Rate During Submaximal Work. Journal of Applied Physiology, 7(2), 218221.

Chau, J. Y., Grunseit, A., Midthjell, K., Holmen, J., Holmen, T. L., Bauman, A. E., \& van der Ploeg, H. P. (2014). Cross-sectional associations of total sitting and leisure screen time with cardiometabolic risk in adults. Results from the HUNT Study, Norway. Journal of Science and Medicine in Sport / Sports Medicine Australia, $17(1)$, 78-84. http://doi.org/10.1016/j.jsams.2013.03.004

Davies, C. a, Vandelanotte, C., Duncan, M. J., \& Uffelen, J. G. Z. V. (2012). Associations of physical activity and screen-time on health related quality of life in adults. Preventive Medicine, 55(1), 46-9. http://doi.org/10.1016/j.ypmed.2012.05.003

Drewnowski, A., Mennella, J. a., Johnson, S. L., \& Bellisle, F. (2012). Sweetness and Food Preference. Journal of Nutrition, 142(7), 1142S1148S. http://doi.org/10.3945/jn.111.149575

Gama, Z. A. da S., Medeiros, C. A. de S., Dantas, A. V. R., \& Souza, T. O. de. (2007). Influência da freqüência de alongamento utilizando facilitação neuromuscular proprioceptiva na flexibilidade dos músculos isquiotibiais. Revista Brasileira de Medicina Do Esporte, 13(1), 33-38. http://doi.org/10.1590/S151786922007000100008

Garber, C. E., Blissmer, B., Deschenes, M. R., Franklin, B. a, Lamonte, M. J., Lee, I.-M., ... Swain, D. P. (2011). American College of Sports Medicine position stand. Quantity and quality of exercise for developing and maintaining cardiorespiratory, musculoskeletal, and neuromotor fitness in apparently healthy adults: guidance for prescribing exercise. Medicine and Science in Sports and Exercise, 43(7), 1334-59. http://doi.org/10.1249/MSS.0b013e318213fefb 
Graves, L. E., Ridgers, N. D., Atkinson, G., \& Atkinson, G. (2010). The effect of active video gaming on children's physical activity, behavior preferences and body composition. Pediatric Exercise Science, 22(4), 535-546.

Grilo, C. M. (1994). Physical activity and obesity. Biomed Pharmacother, 48(3-4), 127-136.

Grøntved, A., Ried-Larsen, M., Møller, N. C., Kristensen, P. L., Wedderkopp, N., Froberg, K., ... Andersen, L. B. (2014). Youth screen-time behaviour is associated with cardiovascular risk in young adulthood: the European Youth Heart Study. European Journal of Preventive Cardiology, 21(1), 49-56.

Haskell, W. L., Lee, I.-M., Pate, R. R., Powell, K. E., Blair, S. N., Franklin, B. a, ... Bauman, A. (2007). Physical activity and public health: updated recommendation for adults from the American College of Sports Medicine and the American Heart Association. Circulation, 116(9), 1081-93. http://doi.org/10.1161/CIRCULATIONAHA.10 7.185649

Heyward, V. H. (2004). Avaliação Física e Prescrição de Exercício: técnicas avançadas. Porto Alegre: Artmed.

Lee, S., \& Shin, S. (2013). Effectiveness of virtual reality using video gaming technology in elderly adults with diabetes mellitus. Diabetes Technology \& Therapeutics, 15(6), 489-96. http://doi.org/10.1089/dia.2013.0050

Lieberman, D. a, Chamberlin, B., Medina, E., Franklin, B. a, Sanner, B. M., \& Vafiadis, D. K. (2011). The power of play: Innovations in Getting Active Summit 2011: a science panel proceedings report from the American Heart Association. Circulation, 123(21), 2507-16. http://doi.org/10.1161/CIR.0b013e318219661d

Lohman, T. G., Ring, K., Pfeiffer, K., Camhi, S., Pratt, C., Pate, R., \& Webber, L. S. (2008). Relationships among fitness, body composition, and physical activity. Medicine and Science in Sport and Exercise, 40(6), 1163-1170. http://doi.org/10.1249/MSS.0b013e318165c86 b.Relationships

Machado, C. H., Silva, A., Horta, P. M., Lopes, A. C. S., \& Santos, L. C. dos. (2013). Efetividade de uma intervenção nutricional associada à prática de atividade física. Caderno de Saúde Coletiva, 21(2), 148-153.

Maddison, R., Foley, L., Mhurchu, C. N., Jiang, Y., Jull, A., Prapavessis, H., ... Rodgers, A. (2011). Effects of active video games on body composition : a randomized controlled trial. The American Journal of Clinical Nutrition, 94(6), 156-163.
http://doi.org/10.3945/ajcn.110.009142.INTRO DUCTION

Maior, A. S., \& Alves, A. (2003). A contribuição dos fatores neurais em fases iniciais do treinamento de força muscular: uma revisão bibliográfica. Revista Motriz, 9(3), 161-168.

Meirelles, C. D. M., \& Gomes, P. S. C. (2004). Efeitos agudos da atividade contra-resistência sobre o gasto energético: revisitando o impacto das principais variáveis. Revista Brasileira de Medicina Do Esporte, 10(21), 122-130. http://doi.org/10.1590/S151786922004000200006

Mendonça, C. P., \& Anjos, L. A. (2004). Aspectos das práticas alimentares e da atividade física como determinantes do crescimento do sobrepeso. Cad. Saúde Pública, 20(3), 698-709.

Pate, R. R. (1988). The Evolving Definition of Physical Fitness. Quest, 40(3), 174-179. http://doi.org/10.1080/00336297.1988.104838 98

Peng, W., Crouse, J. C., \& Lin, J.-H. (2012). Using active video games for physical activity promotion: a systematic review of the current state of research. Health Education \& Behavior: The Official Publication of the Society for Public Health Education, 40(2), 171-92. http://doi.org/10.1177/1090198112444956

Pollock, M. L., \& Wilmore, J. H. (1993). Exercícios na Saúde e na Doença: Avaliação e Prescrição para Reabilitação. Rio de Janeiro: Medsi.

Rodrigues, B. S., Pagnussat, A. de S., \& Chiquetti, E. M. dos S. (2012). Efeitos da Realidade Virtual em Paciente Adulto eom Paralisia Braquial Obstétrica. Revista Neurociencias, 20(4), 567575. http://doi.org/10.4181/RNC.2012.20.720.9p

Santos, C. F. Dos, \& Domingues, C. A. (2008). Avaliação pré e pós-mobilização neural para ganho de ADM em flexão do quadril por meio do alongamento dos isquiotibiais. ConScientiae Saúde, 7(4), 487-495. http://doi.org/10.5585/conssaude.v7i4.1389

Stewart, A., Marfell-Jones, M., Olds, T., \& Ridder, de H. (2001). International Society for the Advancement of Kinantropometry. International Standards for Anthropometric Assessment. Australia. Lower Hutt, New Zealand: International Society for the Advancement of Kinanthropometry

Warburton, D. E. R., Bredin, S. S. D., Horita, L. T. L., Zbogar, D., Scott, J. M., Esch, B. T. a, \& Rhodes, R. E. (2007). The health benefits of interactive video game exercise. Applied Physiology, Nutrition, and Metabolism, 32(4), 655-63. http://doi.org/10.1139/H07-038 